\title{
Evolutionary analysis indicates that DNA alkylation damage is a byproduct of cytosine DNA methyltransferase activity
}

\author{
Silvana Rošić $\mathbb{B}^{1,2,7}$, Rachel Amouroux ${ }^{1,2,7}$, Cristina E. Requena ${ }^{1,2,7}$, Ana Gomes ${ }^{1,2}$, Max Emperle ${ }^{3}$, \\ Toni Beltran ${ }^{1,2}$, Jayant K. Rane ${ }^{1,2}$, Sarah Linnettt, ${ }^{1,2}$ Murray E. Selkirk ${ }^{10}{ }^{4}$, Philipp H. Schiffer ${ }^{5}$, \\ Allison J. Bancroft ${ }^{6}$, Richard K. Grencis ${ }^{6}$, Albert Jeltsch $\mathbb{1}^{3}{ }^{3}$, Petra Hajkova $\mathbb{1}^{1,2 \star}$ and Peter Sarkies $\mathbb{B}^{1,2 \star}$
}

Methylation at the 5 position of cytosine in DNA $(5 \mathrm{meC})$ is a key epigenetic mark in eukaryotes. Once introduced, $5 \mathrm{meC}$ can be maintained through DNA replication by the activity of 'maintenance' DNA methyltransferases (DNMTs). Despite their ancient origin, DNA methylation pathways differ widely across animals, such that $5 \mathrm{meC}$ is either confined to transcribed genes or lost altogether in several lineages. We used comparative epigenomics to investigate the evolution of DNA methylation. Although the model nematode Caenorhabditis elegans lacks DNA methylation, more basal nematodes retain cytosine DNA methylation, which is targeted to repeat loci. We found that DNA methylation coevolved with the DNA alkylation repair enzyme ALKB2 across eukaryotes. In addition, we found that DNMTs introduced the toxic lesion 3-methylcytosine into DNA both in vitro and in vivo. Alkylation damage is therefore intrinsically associated with DNMT activity, and this may promote the loss of DNA methylation in many species.

D NA methylation is an important regulatory mechanism in eukaryotes, with important functions such as transposable element (TE) silencing and gene regulation ${ }^{1} .5 \mathrm{meC}$ acts as an epigenetic modification, which, once introduced by de novo methyltransferases (DNMT3a and DNMT3b in mammals), can be maintained through cell division by the activity of maintenance methyltransferases (DNMT1 in mammals) ${ }^{2}$. Both de novo and maintenance methylation are conserved in many species across eukaryotes, including animals, plants and fungi ${ }^{3,4}$. Nevertheless, DNA methylation pathways evolve rapidly in multiple lineages. Levels of DNA methylation vary widely, with many insects displaying sparse DNA methylation that is confined to a subset of transcribed genes $^{5-9}$. Moreover, in many species, including the model organisms Drosophila melanogaster, C. elegans and Saccharomyces cerevisiae, cytosine DNA methylation has been lost altogether ${ }^{5,10}$. The factors driving such rapid evolution of DNA methylation pathways and their targets remain unclear. We investigated the evolution of DNA methylation in the nematode phylum and more widely across eukaryotes. We found that DNA methylation coevolved with DNA repair pathways and with the ALKB2 alkylation repair system in particular. To explain this, we identified a hitherto unknown offtarget effect of DNMTs, in which they introduce alkylation damage into DNA. Indeed, we found that DNMTs are the major endogenous source of the alkylation 3-methylcytosine $(3 \mathrm{meC})$ lesion in cells. We hypothesize that this toxic activity may act to promote the loss of DNA methylation altogether in multiple lineages.

\section{Results}

DNA methylation is conserved in basal nematodes. To study the factors driving the evolution of DNA methylation, we searched for cytosine DNMTs in nematode genomes across the phylum (Fig. 1a). We first used the Pfam core domain to identify potential cytosine DNMTs and then grouped these using phylogenetic analysis with known eukaryotic DNMTs. All of the identified nematode DNMTs are homologs of DNMT1, DNMT2 or DNMT3. DNMT2, which predominantly methylates tRNA ${ }^{3,4,11}$, is the most widespread among nematodes, but has been lost independently in some lineages, including Caenorhabditis, whereas it is conserved in the closely related parasitic nematode Nippostrongylus brasiliensis (clade V; Fig. 1b). Consistent with previous analyses of individual species ${ }^{12,13}$, we found that the cytosine DNMTs DNMT1 and DNMT3 have been retained in early-branching lineages, confirming that they are ancestral to nematodes. DNMT1 and DNMT3 were most likely lost completely in the common ancestor of the Rhabditida group that contains C. elegans (clades III-V; Fig. 1b). Notably, among the nematodes retaining cytosine DNMTs, some nematodes possess both DNMT1 and DNMT3 (Romanomermis culicivorax), whereas some species possess only DNMT1 (Plectus sambesii) or DNMT3 (Trichuris muris and Trichinella spiralis) (Fig. 1b,c). In species in which DNMT3 is the sole identified DNMT (T. spiralis or T. muris), this protein has not adopted any additional domains from DNMT1 (Fig. 1d,e).

To investigate the effect of the presence of various combinations of cytosine methyltransferases, we measured the abundance of cytosine methylation $(5 \mathrm{meC})$ in genomic DNA using ultrasensitive liquid chromatography/mass spectrometry (LC/MS). $5 \mathrm{meC}$ was clearly detectable in all of the species containing DNMT1 or DNMT3 (Fig. 2a). We did not detect any $5 \mathrm{meC}$ in C. briggsae, which does not have DNMTs, and only detected very low levels in $N$. brasiliensis, which only has DNMT2. Notably, R. culicivorax,

${ }^{1} \mathrm{MRC}$ London Institute of Medical Sciences, London, UK. ${ }^{2}$ Institute of Clinical Sciences, Imperial College London, London, UK. ${ }^{3}$ nstitute of Biochemistry, Universität Stuttgart, Stuttgart, Germany. ${ }^{4}$ Department of Life Sciences, Imperial College London, London, UK. ${ }^{5}$ Department of Ecology and Evolution, University College London, London, UK. ' ${ }^{6}$ School of Biological Sciences and Wellcome Trust Centre for Cell Matrix Research, FBMH, MAHSC, University of Manchester, Manchester, UK. ${ }^{7}$ These authors contributed equally: Silvana Rošić, Rachel Amouroux and Cristina E. Requena. *e-mail: petra.hajkova@lms.mrc.ac.uk; psarkies@imperial.ac.uk 


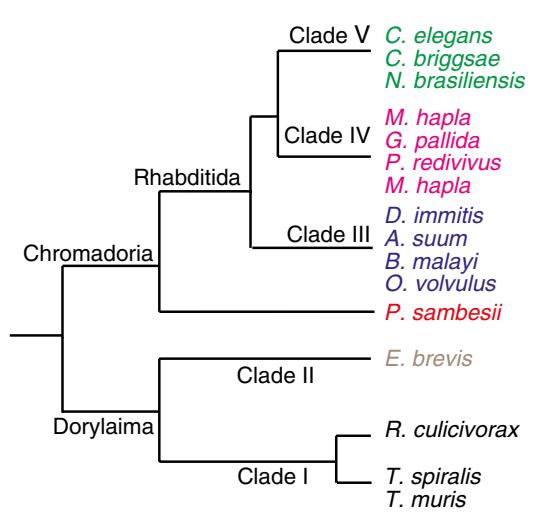

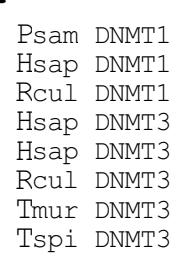

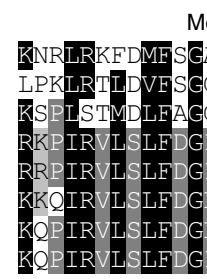

Motif VI (ENV)

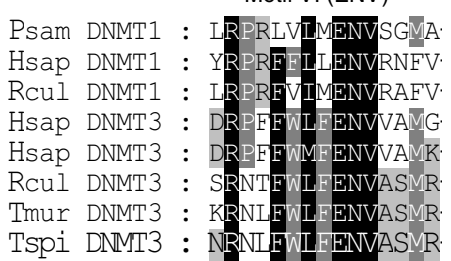

d

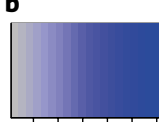
$20 \quad 60 \quad 100$ $-\log _{10}$ (e value)
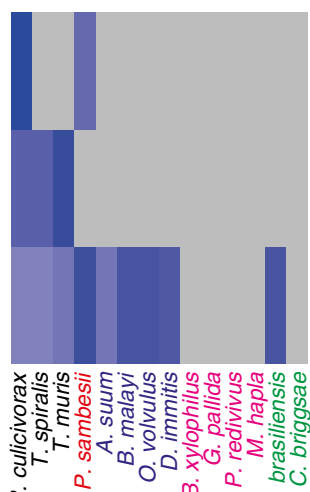

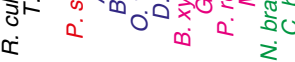

$\begin{array}{llll}\overline{0} & \equiv & \geq & > \\ \frac{\pi}{0} & \frac{0}{0} & \frac{0}{0} & \frac{\pi}{0} \\ \frac{\pi}{0} & \frac{\pi}{0} & \frac{\pi}{0}\end{array}$

DNMT1 DNMT3

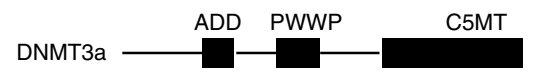

DNMT2

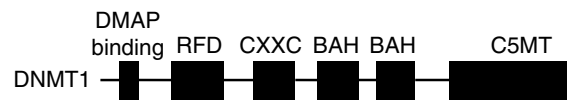

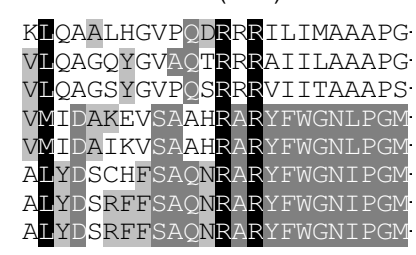

Motif IV (PC[QN])

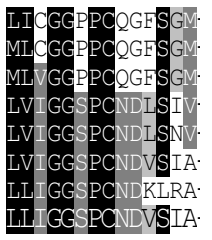

Motif VIII (RXR)

Fig. 1 | Analysis of DNMTs in nematodes. a, Cladogram of nematodes, including the species profiled in this study. Clade nomenclature and phylogenetic positions are taken from ref. ${ }^{33} \cdot \mathbf{b}$. The presence of DNMTs in nematodes as assessed by reciprocal BLAST. Gray indicates no best reciprocal BLAST hit. c, Multiple-sequence alignment showing key motifs important for DNMT activity in nematode DNMTs along with human DNMT1 and DNMT3 for comparison. d, Domains in DNMT1 and DNMT3 from human. e, Conservation of domains in nematode DNMTs as assessed by comparison to the Pfam seed of each domain. Domains from $\mathbf{d}$ that are in the Pfam database are shown, as are those found in at least one nematode DNMT. The N-terminal regions of DNMT1 from $R$. culicivorax and P. sambesii are missing, precluding definitive assessment of the presence or absence of CXXC; this is probably a result of incomplete genome assembly (Methods).

which has both DNMT1 and DNMT3, contained higher levels of genomic $5 \mathrm{meC}$ than the other nematodes.

Nematode DNA methylation is enriched at transposable elements. To investigate the targeting of DNMT1 and DNMT3 to different genomic regions, we carried out whole-genome bisulfite sequencing (Supplementary Table 1). Consistent with our LC/MS analysis (Fig. 2a), we detected significant levels of DNA methylation above the bisulfite non-conversion rate, as estimated based on the inclusion of an unmethylated spike-in in the bisulfite reaction $\left(P<10^{-100}\right.$, Fisher's exact test; Fig. $\left.2 \mathrm{~d}\right)$, in all nematodes with DNMT1 or DNMT3; this methylation was significantly enriched at CG sites over non-CG sites $\left(P<10^{-100}\right.$, Fisher's exact test; Supplementary Fig. 1a). We did not observe significant differences in non-CG methylation between nematodes with just DNMT1 or DNMT3 (Supplementary Fig. 1a). Although we detected trace amounts of $5 \mathrm{meC}$ in $N$. brasiliensis possessing just DNMT2, our bisulfite analysis did not show any significant enrichment of $5 \mathrm{meC}$ above the non-conversion rate (Fig. $2 \mathrm{~d}$ and Supplementary Fig. 1a), suggesting at most a very low and nonspecific activity of DNMT2 on cytosine in DNA, as has been observed in D. melanogaster ${ }^{10}$. Of note, the DNMT1-only methylome and the DNMT3-only methylomes showed different preferences for the nucleotide following the methylation (CG) site, normalized to the abundance of each trinucleotide in the genome. Comparison with bisulfite sequencing data from mouse embryonic stem cells (ESCs) lacking either DNMT1 or DNMT3 ${ }^{14}$ and from the arthropod Bombyx mori, which only has $\mathrm{DNMT}^{8}$, showed that the trinucleotide preferences of DNMT1 were highly similar between nematodes, mammalian cells and B. mori. In contrast, the DNMT3 preferences were different between nematodes and mammalian cells, suggesting differential conservation of DNA interactions for these two types of DNMTs (Fig. 2b,c).

Next, we annotated methylation sites across the entire genome. All of the nematodes with DNMT1 or DNMT3 showed significant enrichment of CG methylation above the genome-wide level $\left(P<10^{-5}\right.$, Fisher's exact test) normalized to CG content for at least one category of repetitive elements (Fig. $2 \mathrm{~d}$ ). In contrast, we did not observe enrichment in overall methylation at genes. Notably, this observation could not be explained by different trinucleotide composition within repeats, as trinucleotide content was similar across different repeat types (Supplementary Fig. 1b,c). C. briggsae (no DNMTs) and N. brasiliensis (DNMT2 only) showed no such enrichment (Fig. 2d). Notably, P. sambesii (DNMT1 only) showed marked enrichment for repeats over the genome-wide background (Fig. 2d). 

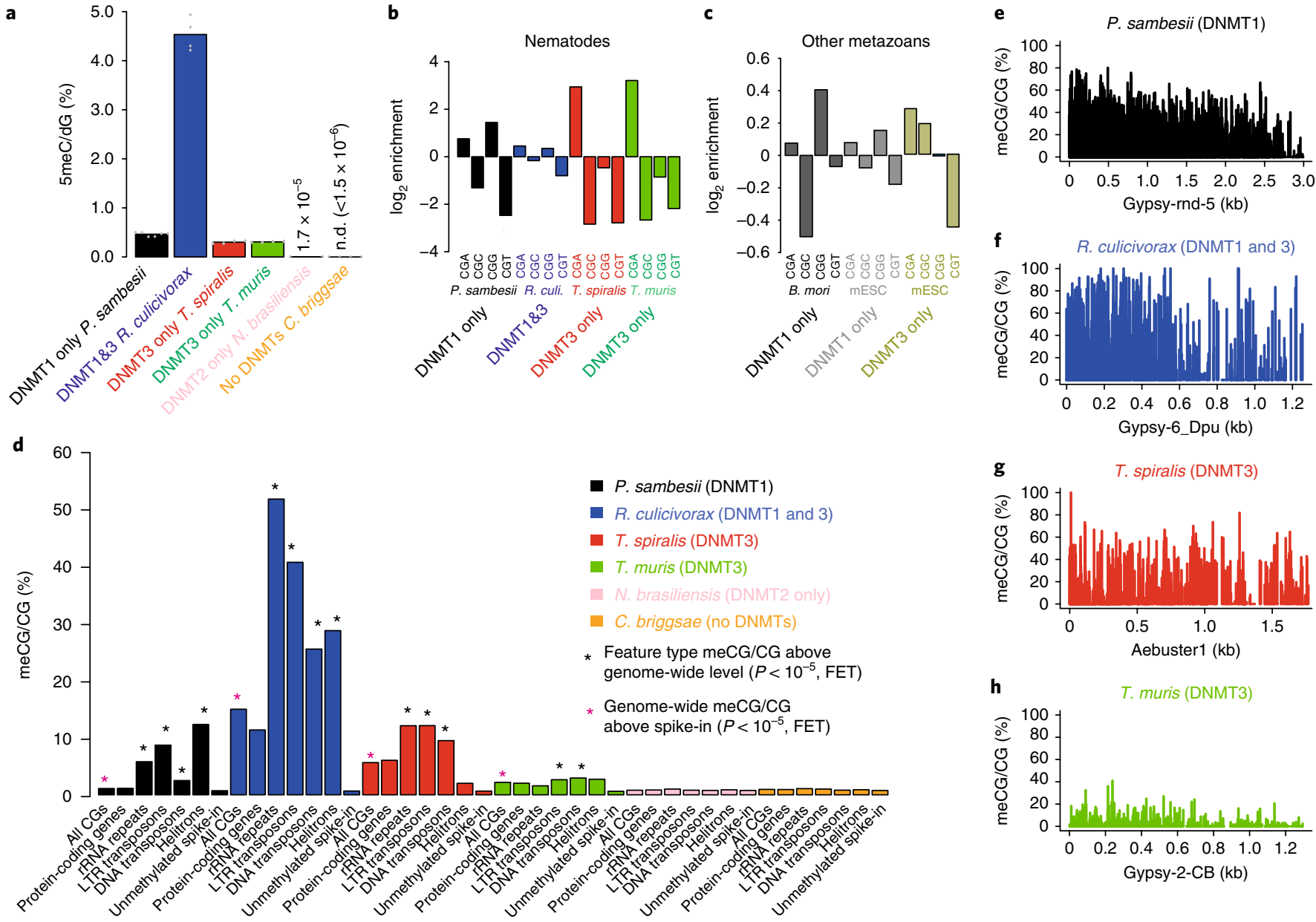

Fig. 2 | Genome-wide DNA methylation analysis of nematodes. a, Quantification of $5 \mathrm{meC}$ in DNA by LC/MS for different nematode species. Bar lines indicate the mean, and error bars represent s.d. Each overlayed point shows the mean of two technical repeats for $n$ independent DNA extractions ( $n=6$, P. sambesii; $n=4$, R. culicivorax, T. spiralis, T. muris, C. briggsae; $n=2$, N. brasiliensis). n.d., not detected. b,c, The overall fraction of sites with $>10 \%$ methylation for each of the specified CG-containing trinucleotides. The total number of CGs analyzed is presented in Supplementary Table 3. d, The average methylation of each CG (meCG) in different annotated regions, as compared with unmethylated spike-in. The total number of CGs analyzed is presented in Supplementary Note 3. FET, Fisher's exact test. e-h, Individual examples of repeat element consensus sequences with high levels of DNA methylation.

DNA methylation could be found across the entire body of many repetitive elements in all species (Fig. 2e-h and Supplementary Figs. 2 and 3) and, genome wide, elements with high levels of methylation were enriched for at least one category of repeats (Fig. 3a-d).

We next examined DNA methylation in protein-coding genes. Analysis of the DNA methylation level across genes revealed that there were no notable populations of genes with higher levels of DNA methylation in P. sambesii (DNMT1 only), T. spiralis (DNMT3 only) or T. muris (DNMT3 only) (Fig. 3e and Supplementary Figs. 2 and 3). The few genes that showed appreciable DNA methylation in these species were likely misannotated repeats, as genes with homology to repeats had higher CG methylation than genes without homology (Supplementary Fig. 4). T. spiralis has been reported to show gene body methylation ${ }^{12}$; however, that study did not normalize for CG content. Given that CG density is markedly higher in the coding regions of all nematodes examined, this likely accounts for the discrepancy with our findings (Supplementary Fig. 5).

In $R$. culicivorax (DNMT1 and DNMT3), there was a bimodal distribution of DNA methylation across genes, with a small population of genes showing elevated DNA methylation (Fig. 3e). This finding is potentially reminiscent of gene body methylation in other invertebrates $^{5-9}$. However, Gene Ontology (GO) analysis of the top 50 methylated genes with GO annotations revealed that $14 \%$ were annotated as nucleic acid integration (enrichment $P=10^{-55}$ compared with all genes, chi-squared test with Benjamani and Hochberg (BH) multiple-test correction; Supplementary Tables 2 and 3); thus, even in R. culicivorax, at least some genes with high levels of methylation may be either misannotated TEs or genes with TE insertions.

Altogether, our analysis of DNA methylation across nematodes indicates that methylation of repeats is its most widely conserved function and was likely to have been present in the common ancestor of nematodes. Methylation in the bodies of transcribed protein-coding genes has been lost altogether in the lineage leading to T. spiralis and T. muris and in P. sambesii, and exists only in a minority of genes in R. culicivorax, and it is therefore not a conserved feature of DNA methylation in nematodes.

It has been argued that gene body methylation is a universal feature of DNMT1 and DNMT3 activity but that repeat-targeted cytosine methylation evolved independently in plants and vertebrates ${ }^{5,6}$. Our data are in accordance with a more nuanced view that the functions of DNA methylation evolve rapidly ${ }^{5,15}$ and that repeat-targeted DNA methylation is found in invertebrates ${ }^{16,17}$. Overall, the rapid evolution of both DNA methylation mechanisms and their targets in nematodes adds to the growing picture of the complex evolution of epigenetic mechanisms in animals ${ }^{5,15,18}$, in 

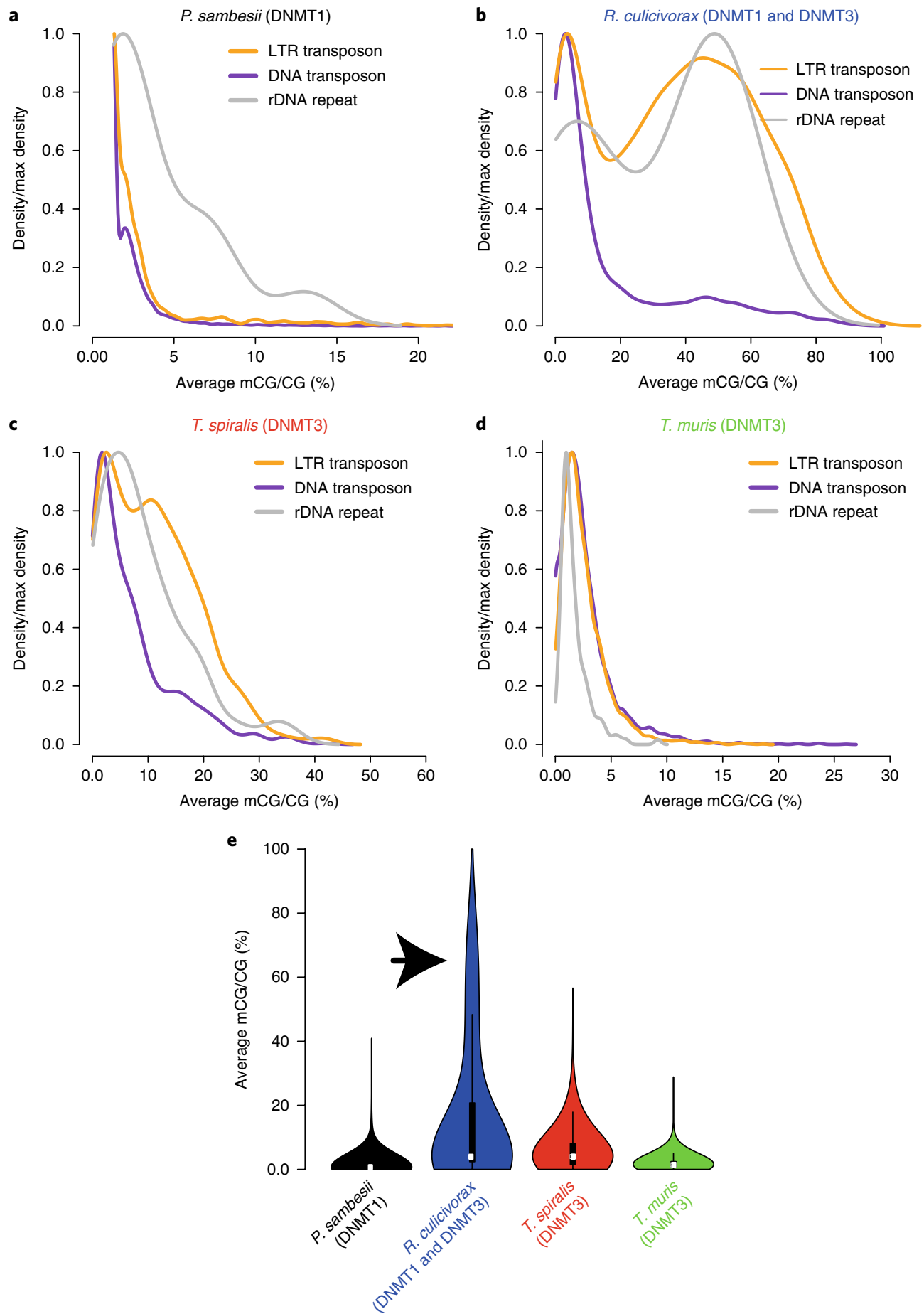

Fig. 3 | Methylation of repeats and gene bodies in nematodes. a-d, Histograms of methylation levels averaged across the body of different genomic features. e, Violin plots of methylation levels of all of the genes across the different species with the subset of genes carrying high levels of DNA methylation observed in $R$. culicivorax indicated by an arrow. The dot is at the median, the box shows interquartile range and the whiskers extend to the greatest point that is no more than 1.5 times the interquartile range.

which the ancestral animals had a rich set of epigenetic mechanisms that have subsequently been lost independently in many descendent organisms.

DNA methylation coevolves with DNA alkylation damage repair across eukaryotes. What drives the rapid evolution of cytosine DNA methylation pathways in animals? One approach to this question is to identify genes coevolving with DNMTs, which may indicate pathways that are linked to the presence or absence of DNA methylation. We analyzed animal genomes in Ensembl (Release 28) and identified 133 human proteins that coevolved with DNMT1 or DNMT3 $(P<0.01$, Fisher's exact test after multiple-test correction; Supplementary Table 4). To our surprise, we found that the most strongly enriched GO term was for DNA repair (Fig. 4a and Supplementary Table 5). In particular, we noted the presence of alkylation repair enzymes among this set (Supplementary Fig. 6 and Supplementary Table 4), including the enzyme ALKB2 and its paralog ALKB3 (a mammalian-specific duplication; hereafter 

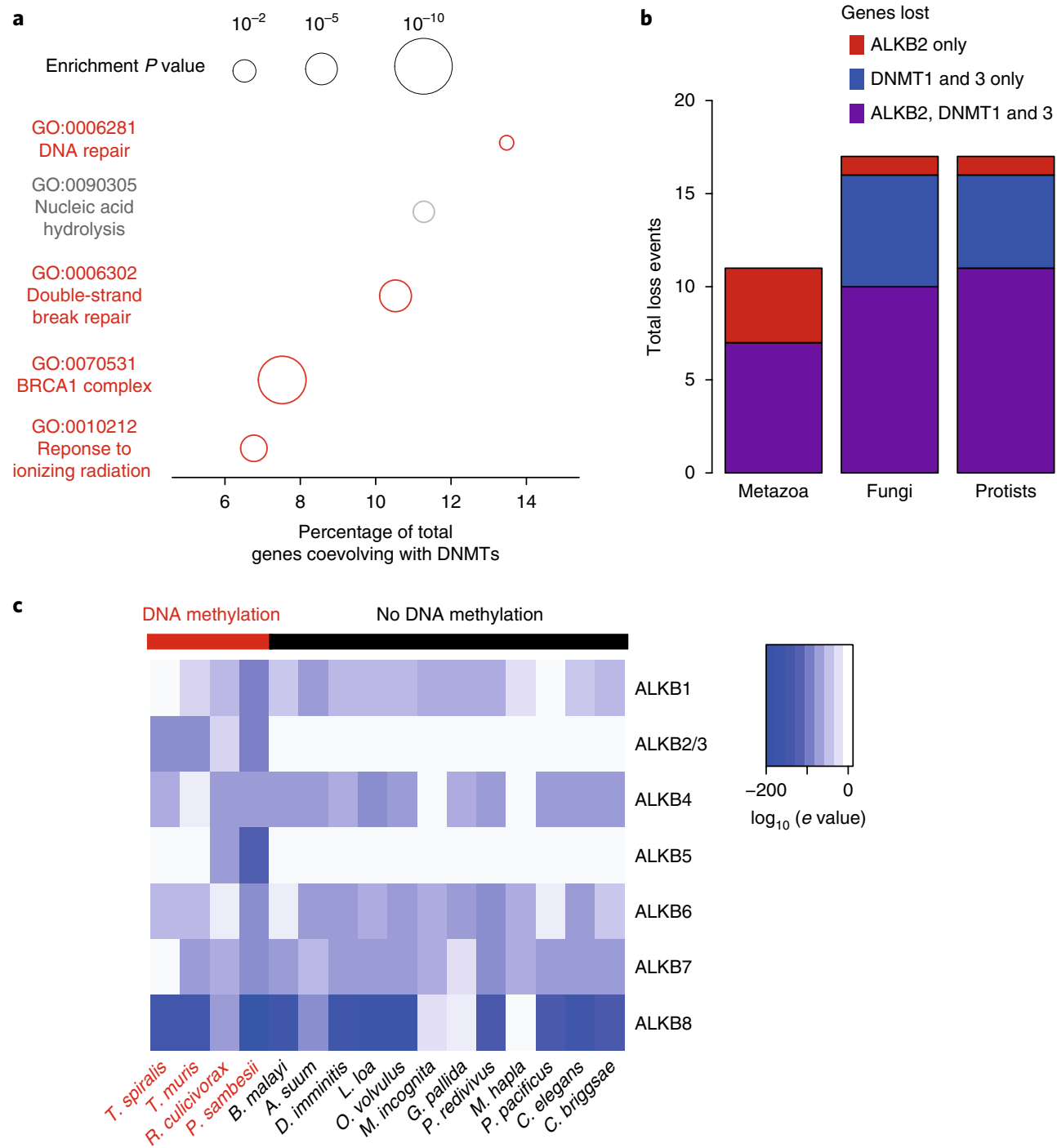

Fig. 4 | Coevolution of DNA methyltransferases with DNA repair enzymes. a, The top five most statistically significantly enriched GO terms in the set of genes that coevolved with DNMTs across animals. Terms associated with DNA repair are shown in red; others are shown in gray. $\mathbf{b}$, The conservation of ALKB2 along with DNMTs across different taxonomic groups. Losses among $n$ independent branches are shown $(n=20$, animals; $n=31$, fungi; $n=23$, protists). c, The conservation of different members of the ALKB family in nematodes with and without DNA methylation.

referred to as ALKB2/3). ALKB2/3 enzymes are members of the $\mathrm{Fe}^{2+}$-dependent oxygenase family of DNA repair enzymes homologous to Escherichia coli $\mathrm{ALKB}^{19}$. Whereas E. coli ALKB repairs a wide range of alkylated adducts, including protein, RNA and DNA, the family has diversified in eukaryotes, with different ALKB family enzymes specializing in the repair of particular substrates. Mammalian ALKB2/3 enzymes are the only members of the ALKB family that repair alkylation damage in $\mathrm{DNA}^{19,20}$ and are the only members that coevolved with the DNMTs DNMT1 and DNMT3 (Fig. 4c and Supplementary Fig. 7). To independently verify the association between DNA methylation and ALKB2/3, we carried out phylogenetic profiling of ALKB2/3 and DNMTs across the eukaryotic genomes in the Ensembl database (fungi, protozoa and animals) and tested for coevolution between ALKB2/3 and DNA methylation. Notably, in this analysis, we corrected for the overrepresentation of several closely related species in Ensembl (for example, the Drosophila genus, in which there are 12 species represented in Ensembl, all of which have no ALKB2/3 and no DNMT1 or DNMT3, or mammals, all of which have ALKB2/3 and DNMT1 and DNMT3) by ensuring that only one member from each lineage with the same profile of ALKB2/3 and DNMTs was included in the analysis (Fig. 4b and Supplementary Figs. 8-11; see Supplementary Tables 6-8 for the list of all of the species considered for the analysis). All three groups showed statistically significant co-occurrence between ALKB2/3 and the presence of at least one cytosine DNMT (DNMT1 and DNMT3) $(P<0.001$ for fungi, $P<0.005$ for animals, $P<0.01$ for protazoa using Fisher's exact test; Fig. $4 \mathrm{~b})$. In addition, in some fungi in which ALKB2/3 are present but DNMT1 is absent, DNMT5, which acts on CG sequences ${ }^{15}$, is conserved (Supplementary Figs. 8-11).

We note that there are some potentially interesting exceptions to the general coevolution between ALKB2/3 and DNMTs, particularly in arthropods, where several species have lost ALKB2/3 while retaining DNMTs. To investigate this further, we compared genomewide methylation levels across arthropods using previously published data from 18 insects ${ }^{9}$, the crustaceans Parhylale hawaiensis ${ }^{17}$, Daphnia pulex and Daphnia magna ${ }^{21}$, and the desert locust Schistocerca gregaria ${ }^{16}$. We found that species retaining ALKB2/3 had $>10$-fold higher median levels of DNA methylation than species that have lost ALKB2/3; this was true in both coding sequences 
<smiles>C[13CH2]Nc1nc(=O)n(C)cc1[Hg]</smiles>

(MRM: 264.1 - 148.1)
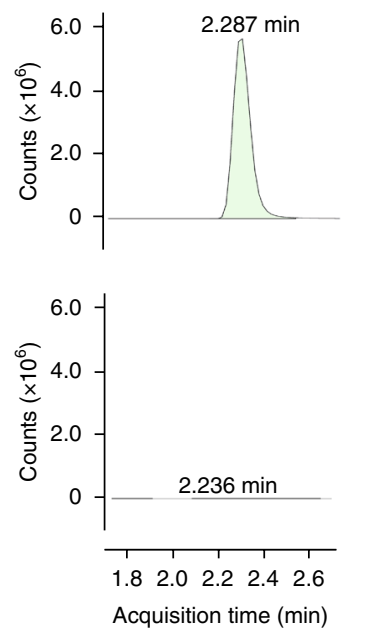

e<smiles>C[13CH2]Nc1nc(=O)n(C)cc1[Hg]</smiles>

(MRM: 264.1 - 148.1)

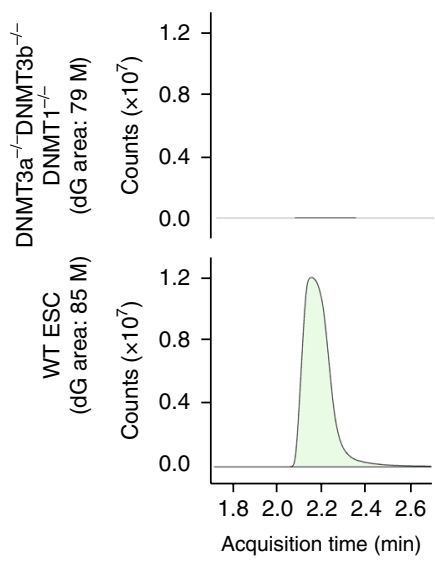

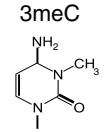

(MRM: 242.1 - 95.1)
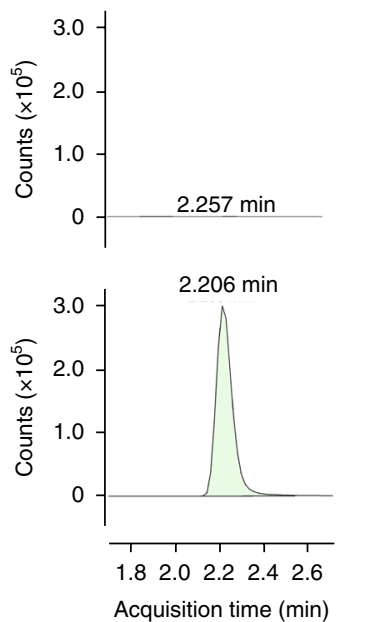<smiles></smiles>

(MRM: 264.1 - 148.1) d<smiles>CN1CCC([Hg])C(=O)N1C</smiles>

(MRM: 242.1 - 95.1)
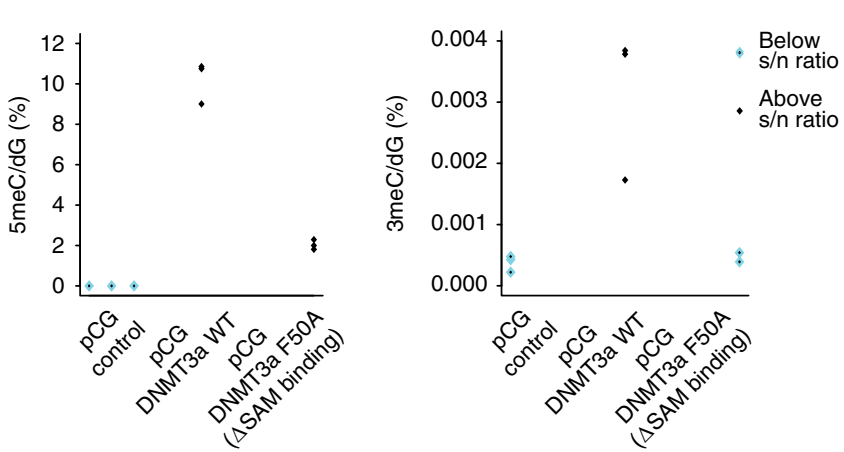

f<smiles>CN1CCC(N)C(=O)N1C</smiles>

(MRM: 242.1 - 95.1)
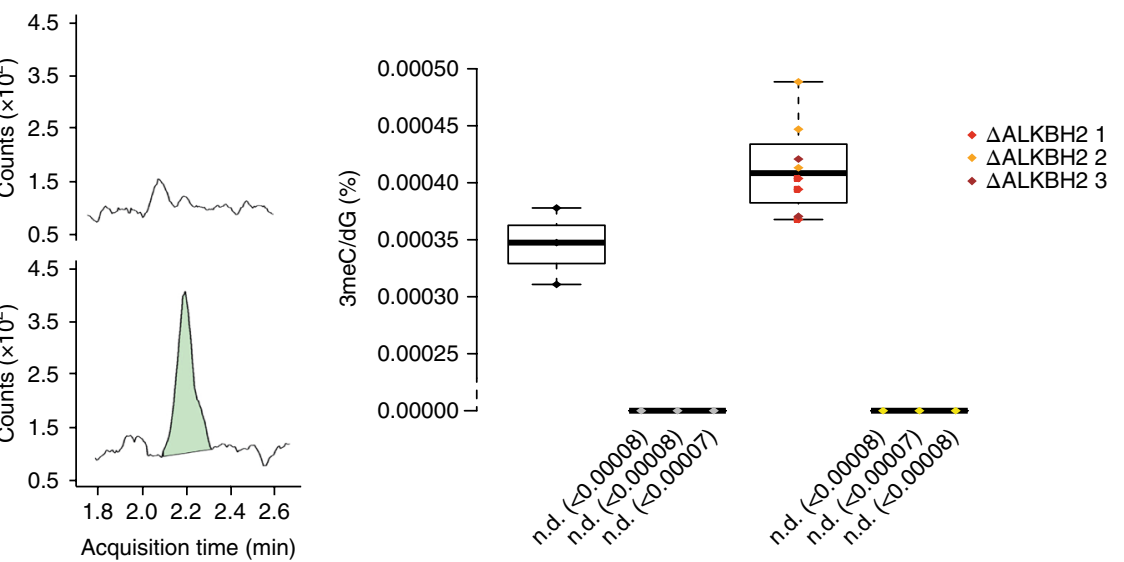

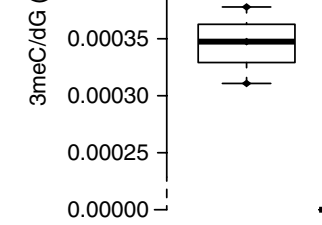

- $\triangle$ ALKBH2 2
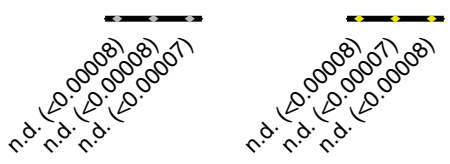

WT

$\Delta \mathrm{DNMT}$
$\mathrm{3a} / 3 \mathrm{~b} / 1$

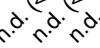

DNMT3a/3b/1 $\triangle \mathrm{ALKBH} 21$

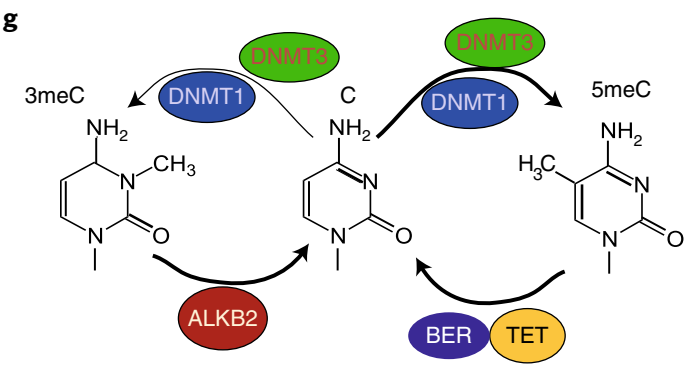

Fig. 5 I DNA alkylation damage in DNA associated with DNMT activity. $\mathbf{a}, \mathbf{b}$, Validation of the method to detect 3 meC specifically in the presence of $5 \mathrm{meC}$ using LC/MS. c,d, LC/MS measurement of $3 \mathrm{meC}$ introduced by the catalytic domain of DNMT3a in vitro compared with $3 \mathrm{meC}$ induction by the F646A mutant, which does not bind the cofactor SAM. Each of the three individual points for each sample shows the mean of two technical replicates for an independent in vitro reaction. Measurements below the signal-to-noise $(\mathrm{s} / \mathrm{n})$ ratio are shown in cyan. e, Example LC/MS traces for $3 \mathrm{meC}$ and $5 \mathrm{meC}$ for ESCs with or without DNMTs. Screenshots of the LC/MS analysis are shown. Colors for peaks are automatically assigned by the software on the basis of the peak settings. $\mathbf{f}, \mathrm{LC} / \mathrm{MS}$ analysis of $3 \mathrm{meC}$ in mouse ESCs with and without DNMTs and ALKBH2 (the Ensembl gene name of the mouse ALKB2 ortholog). The box plots show the interquartile range of $3 \mathrm{meC}$ normalized to $\mathrm{dG}$, with a line at the median and whiskers extending to the furthest point within $95 \%$ of the range. Each of the three points for each cell line shows the mean of two technical replicates for independent DNA extractions.

g, Model for how DNMTs influence methylation on different positions of cytosine. 
and genome wide $(P<0.01$, Wilcoxon unpaired test; Supplementary Fig. 12a,b and Supplementary Table 9).

DNMTs introduce 3meC alkylation damage into DNA. Overall, our analysis confirmed robust and widespread coevolution between ALKB2/3 and DNMTs across eukaryotes. On the basis of this observation, we wondered about a possible mechanistic link between DNA methylation and the presence of alkylation DNA damage. The preferred substrates for ALKB2/3 in DNA are 1-methyladenine $(1 \mathrm{meA})$ and $3 \mathrm{meC}^{22,23}$. We wondered whether the activity of cytosine DNMTs might be associated with the generation of $3 \mathrm{meC}$ in addition to these enzymes producing $5 \mathrm{meC}$. To test this, we used synthetic nucleoside standards to develop an ultrasensitive mass spectroscopy (LC/MS) approach that enabled us to specifically distinguish between and quantify $3 \mathrm{meC}$ and $5 \mathrm{meC}$ in DNA (Fig. 5a,b, Methods and Supplementary Fig. 13a). To further verify this detection method, we treated a plasmid with the mutagen MMS, which, among other lesions, is known to introduce $3 \mathrm{meC}$ into DNA. The $\mathrm{LC} / \mathrm{MS}$ analysis revealed a robust induction of $3 \mathrm{meC}$, but no induction of $5 \mathrm{meC}$ (Supplementary Fig. 13b,c).

To further examine the possible association between DNMTs and $3 \mathrm{meC}$, we tested whether cytosine DNMT activity might be sufficient to produce DNA alkylation damage in vitro. We carried out in vitro methyltransferase reactions using the recombinant catalytic domain of DNMT3a. The subsequent LC/MS analysis identified the robust production of $5 \mathrm{meC}$, as well as clear evidence for $3 \mathrm{meC}$ induction (Fig. 5c,d and Supplementary Fig. 13d,e). The induction of $3 \mathrm{meC}$ was far less abundant and occurred in the ratio 1:2,850 for $3 \mathrm{meC}: 5 \mathrm{meC}$, that is, $3 \mathrm{meC}=\sim 0.035 \%$ of $5 \mathrm{meC}$ (Fig. $5 \mathrm{c}, \mathrm{d}$ ). To verify that this result required the catalytic activity of DNMT3a, we expressed and purified the F646A point mutant of the catalytic domain of DNMT3a, which has a reduced ability to bind the cofactor SAM (Supplementary Fig. 14). Consistent with previous results $^{24}$, we found that this enzyme had markedly reduced catalytic activity in introducing $5 \mathrm{meC}$ (Fig. $5 \mathrm{c}$ ). Notably, this mutation also completely eliminated $3 \mathrm{meC}$ formation, demonstrating that catalytic activity is essential for DNMT3a to promote $3 \mathrm{meC}$ introduction (Fig. 5d). Taken together, these results suggest that DNMTs can use SAM to promote the introduction of $3 \mathrm{meC}$ at a low rate in addition to their usual $5 \mathrm{meC}$ product. Notably, the bacterial methyltransferase $\mathrm{mSSSI}$ also introduced $3 \mathrm{meC}$ in vitro (Supplementary Fig. 13c), suggesting that the introduction of $3 \mathrm{meC}$ may be a general property of cytosine methyltransferases. It is possible that the generation of $3 \mathrm{meC}$ involves a direct catalytic activity of the enzyme; alternatively, DNMTs may promote this indirectly by flipping the base out from the double helix ${ }^{25}$ and positioning it close to SAM.

To test whether DNMTs can promote introduction of $3 \mathrm{meC}$ in vivo, we used our LC/MS method to examine $3 \mathrm{meClevels} \mathrm{in} \mathrm{mouse}$ ESCs carrying DNMT1, DNMT3a and DNMT3b deletions (triple knockout, TKO) ${ }^{26}$. In wild-type (WT) mouse ESCs, we detected a clear signal for $3 \mathrm{meC}$. Notably, the measured $3 \mathrm{meC}$ level was around tenfold lower than the level measured in vitro (Fig. $5 \mathrm{c}-\mathrm{f}$ ), consistent with the existence of endogenous DNA repair mechanisms capable of removing $3 \mathrm{meC}$ (Fig. $5 \mathrm{~g}$ ). In contrast, we were not able to detect any $3 \mathrm{meC}$ in TKO cells $(P=0.0017$, ANOVA; Fig. $5 \mathrm{e}, \mathrm{f})$. As an independent validation, dot blots using an antibody specific for $3 \mathrm{meC}$ showed similar data (Supplementary Fig. 14a,b). We therefore conclude that the presence of active DNMT1 and DNMT3a/b is clearly associated with increased levels of $3 \mathrm{meC}$ in genomic DNA.

Mammalian ALKB2/3 enzymes have been shown to repair $3 \mathrm{meC}$ in vitro and in cultured mouse cells ${ }^{20,22,23}$. To test whether $3 \mathrm{meC}$ induced by DNMT activity is processed by ALKB2 in mouse ESCs, we used the CRISPR-Cas9 system to target deletions to the first exon of Alkb2 in both WT and TKO cells (Supplementary Fig. 15a). We obtained clones with homozygous deletions in both alleles of $A l k b 2$, which showed a reduction in ALKB2 protein, in both
WT and TKO cells (Supplementary Fig. 15b,c). Moreover, these clones showed increased sensitivity to the mutagen MMS relative to their parent line $(P=0.042$, ANOVA test for ALKB deletion; Supplementary Fig. 15d), consistent with disruption of ALKB2 function in repairing alkylation DNA damage. We next analyzed $3 \mathrm{meC}$ levels and found that the loss of ALKB2 led to a 15\% increase in steady-state $3 \mathrm{meC}$ levels $(P=0.02$, ANOVA test for ALKB2 deletion; Fig. 5f), implicating ALKB2 in the removal of $3 \mathrm{meC}$. Notably, in TKO cells, even the lack of ALKB2 did not raise the level of $3 \mathrm{meC}$ above the detection limit of our LC/MS quantification (Fig. 5f). Overall, these data are consistent with ALKB2 being involved in the removal of $3 \mathrm{meC}$ associated with the activity of DNMTs in vivo.

The presence of $5 \mathrm{meC}$ in DNA is known to be mutagenic as a result of the deamination of $5 \mathrm{meC}$ to thymine, resulting in the depletion of CG dinucleotides over evolutionary time ${ }^{27,28} .5 \mathrm{meC}$-to-thymine deamination results in a G-T mismatch. However, alkylation damage such as $3 \mathrm{meC}$ poses a much more severe threat, as $3 \mathrm{meC}$ blocks the DNA polymerases involved in normal DNA replication ${ }^{29,30}$. Thus, our finding that $3 \mathrm{meC}$ is produced by DNMTs indicates that DNMT activity may directly cause replication stress in cells. On the basis of the average GC composition of the mouse genome, we calculated that the level of $3 \mathrm{meC}$ that we observe in vivo corresponds to approximately five modified cytosines in every $10^{6}$ base pairs. The most common form of endogenous DNA damage known is the formation of abasic sites through cytosine deamination and subsequent uracil excision, as well as spontaneous depurination ${ }^{20}$. This form of DNA damage has a marked effect on shaping nucleotide frequencies through evolutionary processes ${ }^{28}$. Abasic sites have been measured in cultured cells and tissues, with estimates ranging from 1-20 nucleotides per $10^{6}$ base pairs ${ }^{31}$. Our results indicate that $3 \mathrm{meC}$, introduced by the off-target activity of DNMTs, exists at similar levels as abasic sites and is therefore one of the most abundant forms of spontaneous DNA damage in cells.

\section{Discussion}

Our results reveal that DNA methylation is a rapidly evolving epigenetic system. We found that, although C. elegans and other nematodes lost their DNA methylation system, other nematode species contain combinations of DNMTs homologous to the mammalian DNMT1 and DNMT3 enzymes that install genomic DNA methylation in these species. Furthermore, we found that, at least in nematodes, DNA methylation is primarily targeted to repetitive elements in the genome.

Notably, our evolutionary analysis of DNA methylation highlights an unexpected coevolution between DNA methylation and DNA repair systems. Our data indicate that DNMT activity is associated with the generation of $3 \mathrm{meC}$ both in vitro and in vivo and that ALKB2 demethylase is required to process this type of alkylation damage. We suggest that the relatively high level of endogenous DNA damage introduced by this off-target activity of DNMTs explains why ALKB2/3 enzymes are generally needed in organisms with $5 \mathrm{meC}$ (Fig. $5 \mathrm{~g}$ ). Even in the presence of ALKB2/3, 3meC introduction by DNMTs is likely to pose a threat to genome stability by causing DNA polymerases to stall, leading to the appearance of double-strand DNA breaks. Consistent with this possibility, members of the BRCA complex and RAD18, both of which are important in DNA double-strand break repair ${ }^{32}$, coevolved with DNMTs (Supplementary Fig. 6 and Supplementary Table 5).

Although future investigation into the relationship between DNA methylation and DNA repair may identify additional mechanistic links, our data indicate that the propensity of cytosine DNMTs to induce alkylation damage may be an important factor explaining the frequent independent losses of DNA methylation across different animal groups. Our data provide an important example of how analysis of the evolutionary relationships between proteins can identify previously unknown biochemical mechanisms. 


\section{Methods}

Methods, including statements of data availability and any associated accession codes and references, are available at https://doi. org/10.1038/s41588-018-0061-8.

Received: 22 July 2016; Accepted: 16 January 2018; Published online: 19 February 2018

\section{References}

1. Law, J. A. \& Jacobsen, S. E. Establishing, maintaining and modifying DNA methylation patterns in plants and animals. Nat. Rev. Genet. 11, 204-220 (2010).

2. Holliday, R. Epigenetics: a historical overview. Epigenetics 1, 76-80 (2006).

3. Ponger, L. \& Li, W. H. Evolutionary diversification of DNA methyltransferases in eukaryotic genomes. Mol. Biol. Evol. 22, 1119-1128 (2005).

4. Jurkowski, T. P. \& Jeltsch, A. On the evolutionary origin of eukaryotic DNA methyltransferases and Dnmt2. PLoS ONE 6, e28104 (2011).

5. Zemach, A., McDaniel, I. E., Silva, P. \& Zilberman, D. Genome-wide evolutionary analysis of eukaryotic DNA methylation. Science 328, 916-919 (2010).

6. Feng, S. et al. Conservation and divergence of methylation patterning in plants and animals. Proc. Natl. Acad. Sci. USA 107, 8689-8694 (2010).

7. Lyko, F. et al. The honey bee epigenomes: differential methylation of brain DNA in queens and workers. PLoS Biol. 8, e1000506 (2010).

8. Xiang, H. et al. Single base-resolution methylome of the silkworm reveals a sparse epigenomic map. Nat. Biotechnol. 28, 516-520 (2010).

9. Bewick, A. J., Vogel, K. J., Moore, A. J. \& Schmitz, R. J. Evolution of DNA methylation across insects. Mol. Biol. Evol. 34, 654-665 (2017).

10. Raddatz, G. et al. Dnmt2-dependent methylomes lack defined DNA methylation patterns. Proc. Natl. Acad. Sci. USA 110, 8627-8631 (2013).

11. Goll, M. G. et al. Methylation of tRNA Asp by the DNA methyltransferase homolog Dnmt2. Science 311, 395-398 (2006).

12. Gao, F. et al. Differential DNA methylation in discrete developmental stages of the parasitic nematode Trichinella spiralis. Genome Biol. 13, R100 (2012).

13. Schiffer, P. H. et al. The genome of Romanomermis culicivorax: revealing fundamental changes in the core developmental genetic toolkit in Nematoda. BMC Genomics 14, 923 (2013).

14. Li, Z. et al. Distinct roles of DNMT1-dependent and DNMT1-independent methylation patterns in the genome of mouse embryonic stem cells. Genome Biol. 16, 115 (2015).

15. Huff, J. T. \& Zilberman, D. Dnmt1-independent CG methylation contributes to nucleosome positioning in diverse eukaryotes. Cell 156, 1286-1297 (2014)

16. Falckenhayn, C. et al. Characterization of genome methylation patterns in the desert locust Schistocerca gregaria. J. Exp. Biol. 216, 1423-1429 (2013).

17. Kao, D. et al. The genome of the crustacean Parhyale hawaiensis, a model for animal development, regeneration, immunity and lignocellulose digestion. eLife 5, e20062 (2016).

18. Sarkies, P. et al. Ancient and novel small RNA pathways compensate for the loss of piRNAs in multiple independent nematode lineages. PLoS Biol. 13, e1002061 (2015)

19. Ougland, R., Rognes, T., Klungland, A. \& Larsen, E. Non-homologous functions of the AlkB homologs. J. Mol. Cell Biol. 7, 494-504 (2015).

20. Sedgwick, B. Repairing DNA-methylation damage. Nat. Rev. Mol. Cell Biol. 5 148-157 (2004).

21. Strepetkaite, D. et al. Analysis of DNA methylation and hydroxymethylation in the genome of crustacean Daphnia pulex. Genes 7, 1 (2015).

22. Ringvoll, J. et al. Repair deficient mice reveal $\mathrm{mABH} 2$ as the primary oxidative demethylase for repairing $1 \mathrm{meA}$ and $3 \mathrm{meC}$ lesions in DNA. EMBO J. 25, 2189-2198 (2006)
23. Nay, S. L., Lee, D.-H., Bates, S. E. \& O'Connor, T. R. Alkbh2 protects against lethality and mutation in primary mouse embryonic fibroblasts. DNA Repair 11, 502-510 (2012).

24. Gowher, H. et al. Mutational analysis of the catalytic domain of the murine Dnmt3a DNA-(cytosine C5)-methyltransferase. J. Mol. Biol. 357, 928-941 (2006)

25. Klimasauskas, S., Kumar, S., Roberts, R. J. \& Cheng, X. HhaI methyltransferase flips its target base out of the DNA helix. Cell 76, 357-369 (1994)

26. Tsumura, A. et al. Maintenance of self-renewal ability of mouse embryonic stem cells in the absence of DNA methyltransferases Dnmt1, Dnmt3a and Dnmt3b. Genes Cells 11, 805-814 (2006).

27. Sved, J. \& Bird, A. The expected equilibrium of the $\mathrm{CpG}$ dinucleotide in vertebrate genomes under a mutation model. Proc. Natl. Acad. Sci. USA 87, 4692-4696 (1990).

28. Alexandrov, L. B. et al. Clock-like mutational processes in human somatic cells. Nat. Genet. 47, 1402-1407 (2015).

29. Drabløs, F. et al. Alkylation damage in DNA and RNA-repair mechanisms and medical significance. DNA Repair 3, 1389-1407 (2004).

30. Furrer, A. \& van Loon, B. Handling the 3-methylcytosine lesion by six human DNA polymerases members of the B-, X-and Y-families. Nucleic Acids Res. 42, 553-566 (2014).

31. Chastain, P. D. II et al. Abasic sites preferentially form at regions undergoing DNA replication. FASEB J. 24, 3674-3680 (2010).

32. Shrivastav, M., De Haro, L. P. \& Nickoloff, J. A. Regulation of DNA double-strand break repair pathway choice. Cell Res. 18, 134-147 (2008).

33. Blaxter, M. L. et al. A molecular evolutionary framework for the phylum Nematoda. Nature 392, 71-75 (1998).

\section{Acknowledgements}

We thank H. Leitch and M. Borkowska for invaluable help with mouse ESC culture. We would like to thank M. Merkenschlager, L. Aragon, J. Sale and B. Lehner for helpful comments on the manuscript, M. Blaxter for advice on nematode genomics, and M. Berriman for access to the $N$. brasiliensis draft genome. P.S. is funded by an Imperial College Research Fellowship. Work in the Sarkies and Hajkova laboratories is funded by the Medical Research Council. P.H. is a recipient of the ERC CoG grant "dynamic modifications" and a member of the EMBO Young Investigator Programme. A.J. and M.E. are funded by DFG JE252/10. R.K.G. and A.J.B. are funded by Wellcome Trust grant $083620 \mathrm{Z}$ and Centre grant $203128 / \mathrm{Z} / 16 / \mathrm{Z}$. P.H.S. is funded by the ERC in a grant to Max Telford (ERC-2012-AdG 322790).

\section{Author contributions}

P.S. and P.H. conceived the study. P.S., P.H. and A.J. designed the experiments. DNA extraction and bisulfite sequencing were carried out by S.R. and P.S. P.S. performed bioinformatic and computational analyses. $3 \mathrm{meC}$ analysis by LC/MS was carried out by R.A., C.E.R., S.L. and P.S. ESC CRISPR deletion and analysis was performed by A.G., J.K.R. and P.S. M.E. and A.J. carried out the in vitro DNMT3a analysis. T.B. and P.H.S. performed genome assembly. S.R., M.E.S., R.K.G. and A.J.B. were responsible for nematode culture. P.S., P.H. and A.J. analyzed the data and prepared the manuscript.

\section{Competing interests}

The authors declare no competing financial interests.

\section{Additional information}

Supplementary information is available for this paper at https://doi.org/10.1038/ s41588-018-0061-8.

Reprints and permissions information is available at www.nature.com/reprints. Correspondence and requests for materials should be addressed to P.H. or P.S. Publisher's note: Springer Nature remains neutral with regard to jurisdictional claims in published maps and institutional affiliations. 


\section{Methods}

Nematode collection and DNA isolation. R. culicivorax adults were a gift from C. Kraus (University of Köln) and derived from the culture of E. Platzer (University of California, Riverside). T. spiralis animals were prepared according to standard methodology. T. muris adults were collected using fine forceps from the ceca of SCID mice orally infected $42 \mathrm{~d}$ previously with 400 embryonated eggs.

P. sambesii animals were grown on low-salt agar with semiliquid HB101 at $25^{\circ} \mathrm{C}$. Adults were isolated from mixed-stage cultures by sorting on a COPAS large-particle sorter.

Analysis of DNA methylation sequencing. We assembled a draft $P$. sambesii genome from Illumina short-read sequencing (see below). Other genomes were taken from Wormbase (C. briggsae, WS240), Wormbase Parasite (N. brasiliensis, T. spiralis, T. muris WBPS4) or Nembase (R. culicivorax). Libraries for bisulfite sequencing were prepared using the Pico Methyl-Seq kit (Zymo Research). Bisulfite sequencing reads were mapped using Bismark, using the bowtie 2 option. To obtain the methylation levels for different CG contexts and for different categories of genomic annotation (Fig. 2), we used the Bismark methylation extractor module to convert Bismark alignments into genome-wide coverage files reporting the methylation status. As the Bismark methylation extractor can only operate on a small number of contigs, before alignments, we had to artificially condense all genomes except that of $C$. briggsae (which is already assembled into 6 chromosomes) into ten 'pseudo-contigs' without disrupting the sequence of the contigs themselves. Subsequently, we selected cytosines covered by at least ten reads using a custom Perl script for further analysis. We converted genome coordinates from pseudo-contigs back to the original contigs and annotated individual CG sites according to gene predictions, either our own (P. sambesii) or those taken from Wormbase (WBPS4; WS245), and repeats were annotated using RepeatMasker using the parameters --no-low, --no-is, --species animalia, using Bedtools $^{34}$. We then obtained percentage methylation by summing the methylated reads and the unmethylated reads across all CG sites within different regions.

Statistical enrichment of CG methylation was calculated using the Fisher's exact test comparing the number of methylated sites and the number of unmethylated sites in both of the genomic regions of interest (that is, genes versus the entire genome).

To analyze the distribution across genes and TEs in more detail (Figs. 2d and 3), we again used Bismark to align DNA methylation sequencing data to contigs directly to avoid artifacts potentially caused by joining contigs together in the middle of repeats or genes. We then used MethylExtract to obtain site-specific methylation information and converted the output to bed files using a custom Perl script. Bedtools was used to annotate CG sites as above, and the mean methylation across individual features (for example, repeat, gene, etc.) was calculated by averaging across the fractional methylation at each site with $>10$ reads of coverage within the feature. Features with $\geq 5$ CGs covered were used to draw Figs. 2d and 3. All statistical analyses and graphics generation were performed in the R environment.

Identification of DNMTs in nematode genomes. We searched the predicted proteins from nematode genomes for cytosine methyltransferase domains using Pfam hmm-search with the cytosine-5-methyltransferase domain. All proteins with matches to this domain were extracted. We then used BLAST to compare these against human DNMT1, DNMT2 and DNMT3 to annotate potential methyltransferases. Any proteins that did not match to DNMT1, DNMT2 or DNMT3 were tested against the Uniprot database; this identified them as bacterial contaminants, and they were removed from further analysis. We verified these annotations by phylogenetic analysis: nematode DNMTs along with selected DNMTs from other animals were aligned using MUSCLE, and these alignments were used to construct a phylogenetic tree according to the workflow in Phylogeny. fr. Domains within the nematode DNMTs were identified using Pfam searches with the seeds for PWWP, BAH and the cytosine DNMT domain. We could not find clear evidence for the CXXC domain in any of the nematode DNMTs, but this could be because of poor assembly of the genome in the $\mathrm{N}$ terminus of the protein in $R$. culicivorax and P. sambesii, as in both of these this region falls near to the boundary of contigs.

Coevolution analysis. We used BLAST on ALKB1-ALKB8 to analyze the conservation of ALKB proteins across the nematodes. The $e$-value of the best hit was tabulated. To identify coevolving proteins across animals in Ensembl, we downloaded each predicted proteome from Ensembl (release 28). We ran LBASTP using the human proteome as the query sequence and each predicted proteome as a database, retaining the best BLAST hit. Proteins with a BLAST hit $\log _{10}(e$-value) less than -20 were given a score of 1 and those with a score greater than -20 were given a score of 0 to build a binary conservation matrix. We then used a Fisher's exact test to identify proteins with a significant tendency to be lost or gained with DNMT3 or DNMT1 using the Benjamini and Hochberg multipletest correction. Gene ontology information for the entire human Uniprot database was downloaded using BiomaRt, and significantly enriched categories were identified using a Fisher's exact test following multiple-test correction. To test further for coevolution between presence of ALKB2/3 and DNMTs, we used a modified phylogenetic profiling method. We first used reciprocal BLAST to test for the presence of ALKB2/3 (retaining any hit that was reciprocal to ALKB2 or ALKB3, including examples where the best BLAST hit for ALKB2 was ALKB3 and vice versa), DNMT1 and DNMT3 in all animal, fungal and protozoan genomes downloaded from Ensembl. To ensure we retained data only for phylogenetically independent loss events, we constructed phylogenetic trees for these groups using the references detailed in the supplementary information (Supplementary Note 2). Finally, we mapped loss of ALKB2, DNMT1 and DNMT3 and collated these for each group before testing for co-occurrence of ALKB2 and one or more of DNMT1 or DNMT3 using Fisher's exact test.

Analysis of DNA methylation levels across arthropods. For all species except S. gregaria, we obtained estimates of $\mathrm{mCG} / \mathrm{CG}$ genome wide and at coding sequences directly from the relevant references ${ }^{9,17,21}$. For $S$. gregaria, the published reference ${ }^{16}$ did not report a genome-wide $\mathrm{mCG} / \mathrm{CG}$ estimate as only coding sequences have been sequenced fully in this organism; thus, we used the FastMC algorithm ${ }^{9}$ to estimate genome-wide $\mathrm{mCG} / \mathrm{CG}$ directly from raw sequencing data and calculated the coding sequence mCG/CG methylation level directly from the reference. We searched for conservation of ALKB2 in these species using the reciprocal BLAST method described above.

Dot blot analysis of methylation in genomic DNA samples. DNA was extracted using the Qiagen DNA Blood/Tissue isolation kit and redissolved in distilled water. DNA was diluted 50:50 with freshly prepared $0.2 \mathrm{M} \mathrm{NaOH}$ and heated for $5 \mathrm{~min}$ at $95^{\circ} \mathrm{C}$ to denature. $2 \mu \mathrm{l}$ of DNA was then spotted onto a nitrocellulose membrane and air-dried before cross-linking with a Stratalinker. The membrane was blocked with 5\% milk in Tris-buffered saline (TBS). Anti-3meC (Active Motif) used at a 1:5,000 dilution or anti-5meC (clone 33D10, Abcam or Active Motif) used at a 1:2,500 dilution was added for an overnight incubation in $1 \%$ milk in TBS with $0.1 \%$ Tween-20. The membrane was washed and exposed to appropriate secondary antibody for $2 \mathrm{~h}$ at room temperature $\left(20^{\circ} \mathrm{C}\right)$ before developing with ECL.

A positive control for $3 \mathrm{meC}$ was prepared by incubating poly $(\mathrm{dI}: \mathrm{dC})$ in the presence of $20 \mathrm{mM}$ MMS (Sigma) for $4 \mathrm{~h}$ at $37^{\circ} \mathrm{C}$. Excess MMS was quenched by addition of $0.2 \mathrm{M} \mathrm{NaOH}$ before dot blot analysis.

Positive and negative controls for $5 \mathrm{meC}$, PCR products from the APC promoter made either with $5 \mathrm{meCTP}$ or CTP, were purchased from Active Motif.

LC/MS. $\mathrm{N}^{3}$-methyl-2' -deoxycytidine ( $3 \mathrm{meC}$ ) standards were purchased from ChemGenes; 2 -deoxycytidine $(\mathrm{dC})$ and $2^{\prime}$-deoxyguanosine $(\mathrm{dG})$ were purchased from Berry and Associates; and $\mathrm{C}^{5}$-methyl-2'-deoxycytidine $(5 \mathrm{meC})$ was purchased from CarboSynth. Genomic DNA or synthetic oligonucleotides were digested to nucleosides for a minimum of $9 \mathrm{~h}$ at $37^{\circ} \mathrm{C}$ using a digestion enzymatic mix (a kind gift from NEB). All samples and standard curve points were spiked with a similar amount of isotope-labeled synthetic nucleosides: $100 \mathrm{fmol}$ of $\mathrm{dC}^{*}$ and $\mathrm{dG}^{*}$ purchased from Silantes and $5 \mathrm{fmol}$ of $5 \mathrm{meC}^{*}$ obtained from T. Carell (Center for Integrated Protein Science at the Department of Chemistry, LudwigMaximilians-Universität München). The nucleosides were separated on an Agilent RRHD Eclipse Plus C18 2.1 $\times 100 \mathrm{~mm}, 1.8 \mathrm{~m}$ column by using the HPLC 1290 system (Agilent) and analyzed using the Agilent 6490 triple-quadrupole mass spectrometer. Quantification was carried out in multiple-reaction monitoring mode (MRM) by monitoring the specific transition pairs of $\mathrm{m} / z 250.1 / 134.1$ for $\mathrm{dC}, 290.1 / 174.1$ for $\mathrm{dG}, 264.1 / 148.1$ for $5 \mathrm{mC}$ and $242.2 / 95.1$ for $3 \mathrm{meC}$. To calculate the concentrations of individual nucleosides (for $\mathrm{dC}, \mathrm{dG}$ and $5 \mathrm{meC}$ ), standard curves representing the ratio of the peak response of known amounts of synthetic nucleosides and the peak response of the isotope-labeled nucleosides were generated and used to convert the peak-area values to corresponding concentrations. For $3 \mathrm{meC}$, the concentrations were calculated directly using a standard curve with light nucleosides. The threshold for peak detection was a signal-to-noise ratio (calculated with a peak-to-peak method) above 10, and the limit of quantification (LOQ) was $25 \mathrm{amol}$ for $5 \mathrm{mC}$ and $50 \mathrm{amol}$ for $3 \mathrm{mC}$. Final measurements were normalized by dividing by the $\mathrm{dG}$ level measured for the same sample. The detectable limit was calculated by dividing the minimum detected value by the dG level for each sample.

DNA methylation in vitro. Unmethylated plasmid was prepared from DAM/ $\mathrm{DCM}^{-}$E. coli cells. For mSSSI methylation we used a pUC19 plasmid and, after purification of the plasmid by MaxiPrep (Qiagen), we treated it with mSSSI (NEB) for $1 \mathrm{~h}$ at $37^{\circ} \mathrm{C}$. To induce alkylation damage, we exposed unmethylated pUC19 plasmid to $20 \mathrm{mM}$ MMS (Sigma) for $1 \mathrm{~h}$ at $25^{\circ} \mathrm{C}$ before purification. DNMT3a and mutants thereof were expressed and purified from E. coli cells as described previously ${ }^{35}$. The reaction mixture was incubated for $2 \mathrm{~h}$ at $37^{\circ} \mathrm{C}$, and DNA was purified using phenol-chloroform extraction and analyzed using LC-MS as described above.

Plectus genome sequencing and assembly. We assembled and annotated a genome for $P$. sambesii using Illumina high-throughput sequencing data and using the methods documented in the supplementary information section (Supplementary Note 1). The final genome had a span of $186 \mathrm{Mb}$ and an N50 of 
4,039 bp, comparing well with other nematode genomes used in this study. The genome has been deposited in NCBI (PRJNA390260).

Generation and validation of ALKB2 deletion mutants. We obtained plasmids containing GFP-tagged CRISPR-Cas9 and guide RNAs targeting the first proteincoding exon of $A L K B 2$ from Sigma. We used Lipofectamine transfection to introduce this plasmid into mouse ESCs and, after recovery of cells for $18 \mathrm{~h}$ at $37^{\circ} \mathrm{C}$, we used FACS to sort GFP-positive cells into individual wells of a 96-well plate. We screened the resultant clones for ALKB2 using PCR across the targeted exon searching for apparent size shifts. We then used Sanger sequencing of the PCR products to select clones showing indels in both alleles. We confirmed ALKB2 protein reduction using western blot analysis with anti-ALKBH2, a mouse monoclonal antibody (C-9; Santa Cruz, sc515789; dilution 1:1,000), using a rabbit anti-mouse HRP-conjugated secondary antibody (Abcam, ab6728; dilution $1: 10,000)$. To test sensitivity to MMS, cells were treated with $200 \mathrm{mM}$ MMS for $1 \mathrm{~h}$ before the MMS was washed out. We then sorted single cells using FACS and counted colonies formed after $5 \mathrm{~d}$, comparing to a control treated with $0 \mathrm{mM}$ MMS for each line.

URLs. Gene Expression Omnibus (GEO), http://www.ncbi.nlm.nih.gov/geo/; Wormbase and Wormbase ParaSite, http://www.sanger.ac.uk/science/tools/ wormbase; Ensembl, http://ensemblgenomes.org/; NCBI, https://www.ncbi. nlm.nih.gov/; DNMT annotation (hmmer version 3.1), http://hmmer.org/; BLAST+ (version 2.2.30), https://blast.ncbi.nlm.nih.gov/; phylogenetic tree construction tools, http://www.phylogeny.fr/; Bismark (version 0.14.2), https:// www.bioinformatics.babraham.ac.uk/projects/bismark/; Bowtie2 (version 2.1.0), http://bowtie-bio.sourceforge.net/bowtie2/; Methylextract (version 1.9),
https://github.com/bioinfoUGR/methylextract?files=1; Bedtools (version 2.19.0), http://bedtools.readthedocs.io/en/latest/; R (version 3.1.0), https://www.r-project.org/.

Life Sciences Reporting Summary. Further information on experimental design is available in the Life Sciences Reporting Summary.

Code availability. Phylogenetic tree construction: MUSCLE v3.8.31 for alignment, Gblocks $0.91 \mathrm{~b}$ for curation and PhyML 3.1 for maximum-likelihood phylogeny. Data integration was performed using Bedtools. Coevolution analysis was performed using BLAST+version 2.2.30. All statistical analysis was carried out using R.

Custom Perl scripts (Perl version 5.16) used for intermediate processing of DNA methylation data are available from the authors upon request.

Data availability. Bisulfite sequencing data have been deposited into GEO with accession GSE104339. The P. sambesii genome assembly has been deposited to NCBI (PRJNA390260). Other nematode genomes are available from Wormbase and Wormbase. Animal, fungal and protist genomes are available from Ensembl. The genome of $P$. hawaiensis is available from NCBI.

\section{References}

34. Quinlan, A. R. \& Hall, I. M. BEDTools: a flexible suite of utilities for comparing genomic features. Bioinformatics 26, 841-842 (2010).

35. Emperle, M., Rajavelu, A., Reinhardt, R., Jurkowska, R. Z. \& Jeltsch, A. Cooperative DNA binding and protein/DNA fiber formation increases the activity of the Dnmt3a DNA methyltransferase. J. Biol. Chem. 289, 29602-29613 (2014). 


\section{Life Sciences Reporting Summary}

Nature Research wishes to improve the reproducibility of the work we publish. This form is published with all life science papers and is intended to promote consistency and transparency in reporting. All life sciences submissions use this form; while some list items might not apply to an individual manuscript, all fields must be completed for clarity.

For further information on the points included in this form, see Reporting Life Sciences Research. For further information on Nature Research policies, including our data availability policy, see Authors \& Referees and the Editorial Policy Checklist.

\section{- Experimental design}

\section{Sample size}

Describe how sample size was determined.

2. Data exclusions

Describe any data exclusions.

\section{Replication}

Describe whether the experimental findings were reliably reproduced. All attempts at replication were successful

\section{Randomization}

Describe how samples/organisms/participants were allocated into experimental groups.

\section{Blinding}

Describe whether the investigators were blinded to group allocation No blinding was performed during data collection and/or analysis.

Note: all studies involving animals and/or human research participants must disclose whether blinding and randomization were used.

\section{Statistical parameters}

For all figures and tables that use statistical methods, confirm that the following items are present in relevant figure legends (or the Methods section if additional space is needed).

$\mathrm{n} / \mathrm{a} \mid$ Confirmed

Х The exact sample size $(n)$ for each experimental group/condition, given as a discrete number and unit of measurement (animals, litters, cultures, etc.) A description of how samples were collected, noting whether measurements were taken from distinct samples or whether the same sample was measured repeatedly.

A statement indicating how many times each experiment was replicated

The statistical test(s) used and whether they are one- or two-sided (note: only common tests should be described solely by name; more complex techniques should be described in the Methods section)

Х A description of any assumptions or corrections, such as an adjustment for multiple comparisons

$\square$ The test results (e.g. $p$ values) given as exact values whenever possible and with confidence intervals noted

$\square$ A summary of the descriptive statistics, including central tendency (e.g. median, mean) and variation (e.g. standard deviation, interquartile range)

$\square$ Clearly defined error bars

See the web collection on statistics for biologists for further resources and guidance.

\section{- Software}

Policy information about availability of computer code

\section{Software}

Describe the software used to analyze the data in this study.

DNA methyltransferase annotation: hmmer (version 3.1) freely available 
from hmmer.org/; blast+ (version 2.2.30) freely available from https:// blast.ncbi.nlm.nih.gov; Phylogenetic tree construction: MUSCLE v3.8.31 for alignment, Gblocks 0.91b for curation and PhyML 3.1 for maximum likelihood phylogeny, all provided via www.Phylogeny.fr. Bisulfite alignment and mapping bismark version 0.14.2 freely available from https://www.bioinformatics.babraham.ac.uk/projects/bismark/; bowtie2 (version 2.1.0) freely available from bowtie-bio.sourceforge.net/bowtie2/; Methylextract version 1.9 freely available from https://github.com/ bioinfoUGR/methylextract?files=1. Bedtools (version 2.19.0) was used for data integration; freely available from http://bedtools.readthedocs.io/en/ latest/.

Coevolution analysis: blast+ version 2.2.30

All statistical analysis was carried out using R (version 3.1.0); freely available from https://www.r-project.org/.

Custom perl scripts (Perl version 5.16) used for intermediate processing DNA methylation data are available on request.

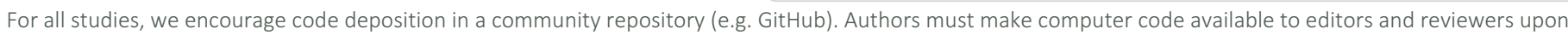
request. The Nature Methods guidance for providing algorithms and software for publication may be useful for any submission.

\section{- Materials and reagents}

Policy information about availability of materials

8. Materials availability

Indicate whether there are restrictions on availability of unique

No restrictions

materials or if these materials are only available for distribution by a for-profit company.

\section{Antibodies}

Describe the antibodies used and how they were validated for use in the system under study (i.e. assay and species).

Mouse monoclonal primary antibody against ALKBH2 (C-9) was purchased from Santa Cruz catalogue number sc-515789. Validation via identification of correct protein size and disappearance of band after disruption of the gene in mouse embryonic stem cells. Secondary antibody was Rabbit antimouse HRP conjugated purchased from Abcam (ab6728). Tested for hybrization against mouse antibodies and not against rabbit antibodies to validate antibody. Anti-3meC used for dot-blots was from Active Motif (61180) and was validated by testing for reactivity with a template treated with MMS for 30 minutes at room temperature and for lack of reactivity with a PCR product made with entirely $5 \mathrm{meC}$.

\section{Eukaryotic cell lines}

a. State the source of each eukaryotic cell line used.

J1S mouse embryonic stem cells from ATCC

TKO mouse embryonic stem cells from Riken RBC

b. Describe the method of cell line authentication used.

PCR was used to validate gene deletions

c. Report whether the cell lines were tested for mycoplasma contamination.

All cell lines tested negative for mycoplasma contaminations repeatedly (c once per month)

d. If any of the cell lines used in the paper are listed in the database of commonly misidentified cell lines maintained by ICLAC, provide a scientific rationale for their use.

No commonly misidentified cell lines were used

\section{- Animals and human research participants}

Policy information about studies involving animals; when reporting animal research, follow the ARRIVE guidelines

\section{Description of research animals}

Provide details on animals and/or animal-derived materials used in the study.

Policy information about studies involving human research participants

\section{Description of human research participants}

Describe the covariate-relevant population characteristics of the human research participants.
No animal or animal-derived material was used

No human research participants 